\title{
Les pistolets Taser, un risque inacceptablement élevé ou suffisamment bas pour être acceptable?
}

\author{
Christine A. Hall, MD, MSc
}

VOIR ARTICLE CONNEXE À LA PAGE 90

ENGLISH VERSION ON PAGE 84

$\mathrm{L}$ es pistolets paralysants Taser, tout comme les armes nucléaires, l'halopéridol, les feux d'artifice, et même les ciseaux, peuvent être dangereux dans les mains de novices. Toutefois, l'interdiction des armes à impulsions électriques (AIÉ), communément appelées pistolets Taser, n'assurera pas nécessairement la sécurité du public. Malgré la controverse entourant l'utilisation du pistolet Taser en Amérique du Nord, la question que nous devrions nous poser au sujet de cette arme ne devrait pas être «Est-ce qu'elle est sûre? », mais plutôt « Est-ce qu'elle est aussi sûre ou plus sûre que les solutions de rechange? »

Dans ce numéro du JCMU, Han et ses collaborateurs ${ }^{1}$ présentent le cas d'un traumatisme oculaire causé par un pistolet Taser. Le traumatisme décrit dans cet article pourrait fort bien raviver la demande incessante et controversée faite par certains concernant l'interdiction des AIÉ comme le pistolet Taser. Mais avant de jeter le bébé avec l'eau du bain, examinons nos options.

Les médecins d'urgence sont bien conscients des difficultés et des risques que présentent les patients violents et instables. À l'instar des policiers, ils ne peuvent pas toujours suivre une progression linéaire méthodique, qui commence par le dialogue et est suivie systématiquement d'un algorithme d'actes de plus en plus agressifs. Le recours à la force policière est similaire à l'assistance respiratoire d'urgence. Il nécessite une évaluation très rapide d'une situation qui souvent se dégrade, la meilleure intervention étant celle qui est la plus susceptible de maitriser complètement la situation tout en réduisant, sans toutefois éliminer, les risques. Tout comme il n'est pas forcément approprié d'utiliser une sonde oropharyngée avant une sonde endotrachéale, il n'est pas nécessairement approprié pour un policier d'avoir recours à la force pour tenter de maîtriser physiquement un patient dans une situation instable ${ }^{2}$.

Maîtriser un individu violent ou désorienté n'est pas chose facile, et le risque imprévisible est fonction de nombreux facteurs liés à l'individu, à l'environnement et à l'intervention. Les effets indésirables, y compris la mort, sont possibles. Dans toute situation dynamique et instable, il faut évaluer le risque par rapport au besoin de maîtriser physiquement la personne, de prévenir les blessures à autrui et de limiter la destruction de la propriété. Même le risque de décès peut être acceptable en présence d'autres dangers immédiats et quantifiables. Pour évaluer ce risque, il faut également connaître les risques associés à d'autres méthodes, y compris les matraques, les armes à impact et les armes à feu, ce qui soulève la question suivante, encore partiellement sans réponse : «Quels sont les risques des AIÉ aux mains des policiers? » et une question encore plus importante : «Est-ce que ces armes sont aussi sûres ou plus sûres que les solutions de rechange? » Si nous ne rendons publics que les résultats négatifs d'une seule méthode de contention, les médias de masse, et même nos revues médicales,

Du département de médecine d'urgence, Autorité sanitaire de l'île de Vancouver, Vancouver (C.-B.); professeure adjointe de clinique, département de chirurgie, Université de la Colombie-Britannique, Vancouver (C.-B.); professeure agrégée, Faculté des Sciences de la santé communautaire, Université de Calgary, Calgary (Alberta).

Les opinions exprimées dans les éditoriaux sont celles des auteurs; elles ne reflètent pas nécessairement celles du JCMU ou de l'Association canadienne des médecins d'urgence.

CJEM 2009;11(1):87-9 
pourraient déformer les faits quant aux risques d'effets indésirables consécutifs à l'usage de la force. On peut exagérer le niveau de risque perçu si l'on ne fait pas une analyse rigoureuse de toutes les AIÉ utilisées (comme arme de contention) dans un contexte de recours à la force.

On a commencé à recueillir des données sur l'utilisation des AIÉ et d'autres méthodes de contention par les policiers au Canada et aux États-Unis, mais la collecte devrait être normalisée et élargie. Des analyses prospectives indépendantes sur les types de blessures ont commencé à tracer le profil des risques du pistolet paralysant Taser, et les premiers rapports sont favorables. Dans un rapport présenté à la 39e Assemblée scientifique du Collège américain des médecins d'urgence en 2007, Bozeman et ses collaborateurs ${ }^{3}$ ont constaté que pour 962 déploiements du pistolet TASER (de TASER International) sur le terrain par 6 organismes américains d'application de la loi, 99,7\% des sujets n'ont subi aucune blessure ou que des blessures légères [intervalle de confiance (IC) à $95 \%$ de 99,1 à 99,9]. Trois personnes ont subi des blessures modérées ou graves ( 1 cas de rhabdomyolyse, 1 de contusion cérébrale et 1 d'hématome épidural). Il y a eu 2 décès en détention, mais les enquêteurs ont déterminé qu'ils n'étaient pas liés à l'utilisation du Taser après qu'ils ont examiné les détails de l'affaire et les résultats des autopsies. Même si ces 2 décès étaient inclus dans le résultat des effets indésirables graves, le pourcentage de sujets sans blessure ou avec blessures légères à la suite du déploiement d'un pistolet Taser sur le terrain était de 99,4\% (IC à $95 \%$ de 98,8 à 99,8$)$ et le pourcentage de blessures modérées ou graves était de $0,5 \%$ (IC à $95 \%$ de 0,2 à 1,2).

De même, au Canada, un examen préliminaire des données du Service de police de Calgary (2005-2007) ${ }^{4}$ révèle que les policiers emploient la force dans seulement $0,07 \%$ (environ 1 pour 1400 ) des interactions face-à-face policepublic et que le déploiement des pistolets Taser contre 271 personnes n'a causé aucun décès ni aucune blessure ou des blessures légères dans 236 cas ( $87 \%$, IC à $95 \%$ de 82,5 à 90,8). Parmi les 35 personnes restantes, 3 ont été hospitalisées (1,1\% de toute la cohorte, IC à $95 \%$ de 0,2 à 3,2 ). L'examen de ces derniers cas est en cours afin de déterminer si le déploiement du pistolet Taser est en cause. Cette étude va bientôt s'élargir pour inclure de nombreux services de police du Canada et des États-Unis.

Auparavant, on a signalé des décès liés à l'usage de moyens de neutralisation physique (par exemple, la technique de contrôle du cou, les pieds et poings liés, la contention par de multiples agents de police) et même le poivre de cayenne. Les décès consécutifs à l'emploi de la force par les policiers ne sont pas nécessairement limités à l'emploi d'AIÉ. Les groupes qui demandent de cesser complètement d'utiliser le pistolet Taser font fi des constatations voulant que l'emploi de cette arme sur le terrain pose un risque très faible. Même les décès qui ont eu lieu à la suite du déploiement du Taser ne peuvent être considérés sans tenir compte d'un contexte plus large. Nous ne comprenons toujours pas encore pleinement le rôle que joue la notion controversée de " délire agité », et le rôle de cause à effet (le cas échéant) que les AIÉ ou les autres moyens de contention tiennent dans les décès liés à l'emploi de la force.

D'aucuns ont suggéré de simplement renoncer à l'usage des AIÉ jusqu'à ce que l'on puisse faire d'autres études sur la sécurité de cette arme ${ }^{5,6}$. Toutefois, de telles études ne peuvent être fondées sur des enquêtes menées auprès de bénévoles en santé ou d'animaux. Les corps policiers devraient continuer d'utiliser le pistolet Taser et recueillir des données rigoureuses chaque fois qu'ils la déploient. Sinon, nous éliminons un moyen de contention raisonnablement sûr sans en apprendre davantage sur le risque relatif d'autres méthodes préjudiciables comme les matraques, les armes à feu ou des méthodes de contention non éprouvées.

En date du 4 novembre 2008, 28 hommes avaient trouvé la mort après l'utilisation par les policiers d'un pistolet Taser (Steve Palmer, Centre canadien de recherches policières, Ottawa, Ontario : communication personnelle, 2008). Or, les rapports de police sur l'emploi de la force au Canada ne sont pas normalisés et ils ne sont pas transmis d'une agence à l'autre. En outre, il n'existe pas de base de données nationale sur les décès en détention. Le manque de données anatomiques concernant l'endroit où se plantent les fléchettes entrave la capacité d'interpréter les études chez l'animal qui suggèrent que l'endroit où pénètrent les fléchettes a une incidence sur le risque d'arythmie ${ }^{7,8}$. Il faut normaliser la consignation d'informations sur les traumatismes et les comportements menant à l'emploi de la force ou les dérogations aux politiques et la verser dans un registre national.

Les chercheurs indépendants de l'industrie doivent avoir accès à des registres nationaux sur l'emploi de la force par les corps policiers. Les services de police et les décideurs seraient plus en mesure d'évaluer objectivement la sécurité de ces armes s'ils avaient accès à des données factuelles et indépendantes de l'information obtenue des fabricants. Une évaluation juste des risques réels limiterait la possibilité que des décisions hâtives soient prises en réaction à des données non scientifiques ou à une mauvaise interprétation de la littérature médicale?.

Une bonne information a un prix, mais le financement doit également être indépendant de l'industrie. Les 
gouvernements et les autres organismes qui recommandent de mener des études devraient financer ces initiatives ${ }^{5,69-11}$. Des subventions provenant de fabricants de AIÉ, peu importe la transparence, ne sont pas une option acceptable, compte tenu des conflits évidents qui existent. Il est également essentiel d'obtenir la participation de médecins intéressés. Nous devons accepter cette responsabilité. La création d'un groupe de travail national formé de médecins que le sujet des blessures et des décès en détention intéresse apporterait ses connaissances au débat et présenterait un point de vue neutre contraire au ton confiant de l'industrie en réponse aux publications sur les AIÉ dans les journaux médicaux ${ }^{12-14}$.

Le pistolet Taser est une arme, et toutes les armes posent des risques. Dans les mains d'une personne ayant reçu la formation appropriée, c'est aussi un outil, et les médecins d'urgence connaissent bien les outils qui comportent des risques. Nous devons demeurer libres de la possibilité d'être partiaux à l'égard de l'industrie et d'alarmer le public. L'heure est venue de mener des études au niveau national sur les AIÉ et les autres méthodes de contention. La sécurité de nos citoyens et de nos agents de police est en cause.

Conflits d'intérêts : La $\mathrm{D}^{\text {re }}$ Hall est la chercheuse principale de l'étude « RESTRAINT », une étude prospective multicentrique sur les personnes contre lesquelles les policiers ont utilisé des moyens de contention pour les immobiliser, et de l'étude «SCAPGAS », qui vise à déterminer si l'on pourrait mettre au point une autre méthode pour obtenir un échantillon de sang capillaire auprès des patients agités, combatifs et souvent menottés en milieu préhospitalier. Le Centre canadien de recherches policières finance ces deux études. La $\mathrm{D}^{\text {re }}$ Hall poursuit également une revue des dossiers des personnes décédées en détention en Alberta, sur une période de 10 ans, avec la coopération du Solliciteur général de l'Alberta et du Bureau du médecin légiste en chef de l'Alberta. Cette étude est financée en partie par le Centre canadien de recherches policières. La D ${ }^{\text {re }}$ Hall n'a aucun lien financier ou autre avec Taser International.

\section{Références bibliographiques}

1. Han JS, Chopra A, Carr C. Ophthalmic injuries from a TASER. CJEM 2009;11:90-3.

2. Canadian Association of Chiefs of Police, Board of Directors. A national use of force framework. Ottawa $(\mathrm{ON})$ : The Association; 2000. Disponible sur : http://ilecnet.org/Standards/Canada $\% 20$ National\%20Use\%20of\%20Force\%20Model\%202000.pdf (consulté le 24 novembre 2008).

3. Bozeman WP, Winslow JE, Hauda WE II, et al. Injury profile of TASER electrical conducted energy weapons (CEWs). 39th Scientific Assembly of the American College of Emergency Physicians. Seattle (WA): Oct 8-11, 2007.

4. Butler C. Force Science News \# 102. Mankato (MN): Force Science Research Center; 2008. Disponible sur : www.force sciencenews.com/home/current.html (consulté le 24 novembre 2008).

5. Amnesty International. Amnesty International's continuing concerns about taser use. London (UK): Amnesty International; 2008. Disponible sur : www.amnesty.org/en/library/asset /AMR51/030/2006/en/dom-AMR510302006en.pdf (consulté le 24 novembre 2008 ).

6. American Civil Liberties Union. Citizen deaths in police custody, ACLU of Colorado call for limits on use of electroshock weapons. New York (NY): The Union; 2004. Disponible sur : www.aclu.org/crimjustice/gen/10119prs20040226.html (consulté le 24 novembre 2008).

7. Nanthakumar K, Billingsley IM, Masse S, et al. Cardiac electrophysiological consequences of neuromuscular incapacitating device discharges. J Am Coll Cardiol 2006;48:798-804.

8. Dennis AJ, Valentino DJ, Walter RJ, et al. Acute effects of Taser X26: discharges in a swine model. J Trauma 2007;63:581-9.

9. Kiedrowski J, Petrunik M, Melchers R. Examen indépendant de l'adoption de l'arme à impulsions et de son utilisation par la Gendarmerie royale du Canda. Ottawa, Ontario : Gendarmerie royale du Canda; 2008. Disponible sur : www.rcmp-grc.gc .ca/ccaps/cew/kiedrowski_report_f.htm (consulté le 24 novembre 2008).

10. Communiqué de la Commission d'enquête Braidwood. Victoria, C.-B. : Province de la C.-B.; 2008. www.braidwoodinquiry.ca/

11. Breitkreuz G. Rapport du Comité permanent de la sécurité publique et nationale : Étude sur l'arme à impulsions électriques TaserMD. Ottawa, Ontario. Chambre des communes Canada; 2008. Disponible sur : www2.parl.gc.ca/Content/HOC /Committee/392/SECU/Reports/RP3582906/392_SECU_Rpt04 _PDF/392_SECU_Rpt04-f.pdf (consulté le 24 novembre 2008).

12. Luceri RM. An open letter to the law enforcement community. Florida Arrhythmia Consultants. J Am Coll Cardiol 2007;49:732.

13. Kroll MW, Calkins H, Luceri RM. Electronic control devices and the clinical milieu. J Am Coll Cardiol 2007;49:732-3.

14. Kroll MW, Calkins H, Luceri RM, et al. Electronic control devices. CMAJ 2008;179:342-3.

Correspondance : $\mathrm{D}^{\text {re }}$ Christine Hall, Vancouver Island Research, 1952, rue Bay, Pavillon Memorial, aile 1 Kenning, Victoria BC V8R 1J8; chris.hall@cprc.org 